\title{
Road Slope Analysis and Filtering for Driveline Shuffle Simulation
}

\author{
Andreas Myklebust* Lars Eriksson* \\ * Vehicular Systems, Department of Electrical Engineering, Linköping \\ University, 58183 Linköping Sweden (e-mail: andmy, \\ larer@isy.liu.se).
}

\begin{abstract}
In powertrain analysis, simulation of driveline models are standard tools, where efficient and accurate simulations are important features of the models. One input signal with high impact on the accuracy is the road slope. Here it is found that the amplitude discretization in production road-slope sensors can excite vehicle shuffle dynamics in the model, which is not present in the real vehicle. To overcome this problem road-slope information is analyzed with the aid of both measured and synthetic road profiles, where the latter are generated from regulatory road specifications. The analysis shows that it is possible to separate vehicle shuffle resonances and road-slope information, and designs are proposed for on- and off-line filtering of the road-slope-sensor signal in spatial coordinates. Applying the filter to measured data shows that vehicle shuffle is significantly attenuated, while the shape of the road slope profile is maintained. As a byproduct the use of smoothing the rolling resistance is shown.
\end{abstract}

Keywords: road profile, discretization noise, frequency content

\section{INTRODUCTION}

When analyzing powertrains and their controls, the results will of course depend on the model but also on the input signals. The quality of the input signals have a big impact on simulation results, e.g. noise components in the inputs can have a profound effect on the outputs. A motivating example is given in Figure 3 where powertrain oscillations are induced due to sensor discretization levels, of integer percentage points, in road slope.

This example of discretization noise in the road-slope signal and how it influences simulated dynamics of a driveline is in focus here. Attention is also given to how a measured road-slope-sensor signal can be filtered so that the high frequency content, that can excite driveline dynamics and might result in vehicle shuffle, is removed while the relevant information is maintained. This is of importance when powertrains are simulated for control applications where vehicle shuffle has to be avoided. Examples of such control applications are vehicle-speed control, (Pettersson and Nielsen, 2002), driver-filter design, (Gerhardt et al., 1998), gear-shift control, (Fredriksson and Egardt, 2003), (Pettersson and Nielsen, 2000), and launch control, (Garofalo et al., 2002), (Dolcini et al., 2008).

Little work exists in the area of analyzing and filtering road slope data, even though road-grade information is used in different applications. For example Gao et al. (2011) studies road slope estimation and also how an incorrect estimation of the constant road slope effects the control of clutch disengagement. Estimation of the road slope for various applications is also treated in e.g. Lingman and Schmidtbauer (2002), Sebsadji et al. (2008) and Bae et al.

\footnotetext{
* Vinnova industrial excellence center LINK-SIC and Scania CV have supported this work.
}

(2001). In Sahlholm (2011), where a road slope estimator is developed, low frequency errors are introduced to study the effect on look-ahead control and motivate the need of a good road grade estimator. Sahlholm (2011) filtered the road data using a third-order Butterworth low-pass filter with spatial cut-off frequency of $9 \cdot 10^{-3} \mathrm{~m}^{-1}$ before being used. In the related literature there is no quantitative analysis of the frequency content in the road-slope data.

A driveline model is developed for analysis of the interaction between road-slope data and vehicle shuffle. Measurement data is collected from a heavy duty truck, in order to both validate the model and to get realistic signals for the study. In addition the frequency content of different roads is analyzed, both theoretically and through measured road-slope data.

\section{POWERTRAIN MODEL}

In order to investigate how the quality of the road-slope signal affects simulation, a simulation model is required. A longitudinal model of a heavy-duty truck has therefore been developed. The model has to capture important dynamics in the driveline and how they make the truck shuffle. The model is an extension of the model in Pettersson (1997) that was used for evaluation of oscillation damping by engine control during tip-in maneuvers. Pettersson (1997) identified the important flexibilities in the driveline that cause vehicle shuffle and therefore the same model structure is used here. The model is built in a modular way and then condensed into a state space model.

An overview of the different modules and their information exchange is given in Figure 1, which also defines the nomenclature used in this paper. Each module corresponds to a part in the powertrain, Internal Combustion Engine 
(ICE), clutch, gearbox, propeller shaft, final drive, drive shafts and vehicle dynamics.

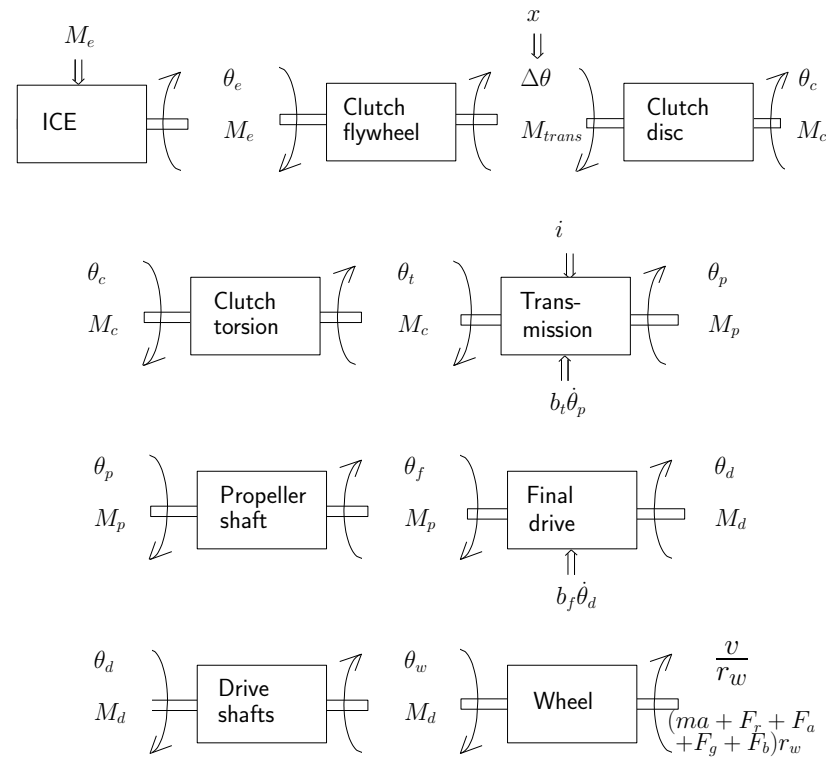

Fig. 1. Sketch of the subsystems and their information exchange in the truck model.

\subsection{Internal Combustion Engine}

The ICE produces the engine torque, $M_{e}$, that is given as model input. Note that this is the net (brake) torque of the ICE, e.g. $M_{e}=0$ with open clutch will keep the engine speed constant.

\subsection{Clutch}

The clutch is modeled in three separate parts. The actuator, the clutch disc where slip might occur, and a torsional part that is flexible.

Clutch Actuator The clutch position, $x$, is normalized by its maximum stroke, $x_{\max }$ to get a relative position. The relative position is assumed to have an effective range, $x_{\mathrm{eff}}$ where the resulting normal force between the clutch plates are unsaturated. This effective range is transformed so that 0 corresponds to zero normal force and 1 corresponds to maximum normal force. This transformed signal is assumed to be proportional to the applied normal force.

The natural output from the actuator is the clamping force, $F_{N}$. Nevertheless the clamping force is directly recalculated into a transmittable torque, $M_{\text {trans }}=k \mu F_{N}$, that is used as actuator output. There are two friction coefficients, one static, $\mu_{s}$, and one dynamic, $\mu_{k}$. Let $\mu_{k}$ be part of $k_{\text {clutch }}$ and define the ratio of the friction coefficients, $k_{\mu}=\frac{\mu_{s}}{\mu_{k}}$. Then the equations for the clutch actuator become:

$$
x_{\mathrm{eff}}= \begin{cases}L_{l}, & \frac{x}{x_{\max }}<L_{l} \\ \frac{x}{x_{\max }}, & L_{l} \leq \frac{x}{x_{\max }} \leq L_{u} \\ L_{u}, & L_{u}<\frac{x}{x_{\max }}\end{cases}
$$

$$
\begin{gathered}
M_{\text {trans }, k}=k_{\text {clutch }} \frac{L_{u}-x_{\text {eff }}}{L_{u}-L_{l}} \\
M_{\text {trans }, s}=k_{\mu} M_{\text {trans }, k}
\end{gathered}
$$

Note that (2) is a simple model of the slipping clutch compared to Myklebust and Eriksson (2012).

Clutch Disc The modeled clutch is a single-plate dry clutch with two contact surfaces. Torque is transferred between the clutch disc and flywheel through friction. The friction is modeled as coulomb friction with stick-slip behavior. Torque from the ICE, $M_{e}$, and driveline, $M_{c}$, are inputs and angular velocity \& angle on both sides, $\dot{\theta}_{e} \&$ $\theta_{e}$ and $\dot{\theta}_{c} \& \theta_{c}$, respectively, are outputs.

The clutch model has two modes, locked and slipping mode. While in locked mode, the clutch behaves as one rigid body, whereas during slipping the clutch consists of two bodies where each one has an angular velocity and position. The condition for the transition from slipping to locked mode is that the speed difference is zero and the transmitted torque, $M_{\text {trans }}$ is less than the static transmittable torque, $M_{\text {trans,s }}$. The condition for transition from locked to slipping mode is when $M_{\text {trans }}$ rises above $M_{\text {trans,s }}$.

Using $\theta_{e}, \dot{\theta}_{e}, \theta_{c}$, and $\dot{\theta}_{c}$ as states gives the following equations for the clutch disc:

Conditions for switching from slipping to locked mode:

$$
\begin{aligned}
\dot{\theta}_{e} & =\dot{\theta}_{c} \\
M_{\text {trans }} & \leq M_{\text {trans,s }}
\end{aligned}
$$

Equations for the clutch in locked mode:

$$
\begin{aligned}
M_{e}-M_{c} & =\left(J_{\mathrm{ICE}}+J_{\mathrm{fw}}+J_{c}\right) \ddot{\theta}_{e} \\
\dot{\theta}_{c} & =\dot{\theta}_{e} \\
M_{\mathrm{trans}} & =\frac{M_{e} J_{c}+M_{c}\left(J_{e}+J_{\mathrm{fw}}\right)}{J_{e}+J_{\mathrm{fw}}+J_{c}}
\end{aligned}
$$

Conditions for switching from locked to slipping mode:

$$
M_{\text {trans }} \geq M_{\text {trans,s }}
$$

Equations specific to the clutch in slipping mode:

$$
\begin{aligned}
M_{\mathrm{trans}} & =\operatorname{sgn}\left(\dot{\theta}_{e}-\dot{\theta}_{c}\right) M_{\mathrm{trans}, \mathrm{k}} \\
M_{e}-M_{\mathrm{trans}} & =\left(J_{e}+J_{\mathrm{fw}}\right) \ddot{\theta}_{e} \\
M_{\mathrm{trans}}-M_{c} & =J_{c} \ddot{\theta}_{c}
\end{aligned}
$$

Torsional Part The main flexibility of the clutch is in the torsion springs in the clutch disc. They are located on the vehicle side of the friction surfaces. Therefore the flexibility of the clutch is modeled as a separate part of the clutch, located on the transmission side of the clutch disc, like in Moon et al. (2004).

The clutch torsional part is modeled as a torsional spring and damper. It takes speed \& angle from the clutch disc, $\dot{\theta}_{c} \& \theta_{c}$, and the input shaft of the transmission, $\dot{\theta}_{t} \& \theta_{t}$, as input and returns a torque, $M_{c}$. The equation for the torsional part is:

$$
M_{c}=c_{c}\left(\dot{\theta}_{c}-\dot{\theta}_{t}\right)+k_{c}\left(\theta_{c}-\theta_{t}\right)
$$




\subsection{Transmission}

The gear number (input) is converted to a gear ratio, $i_{t, i}$ and gearbox inertia, $J_{t, i}$. The gear ratio scales the inputside torque, $M_{c}$, \& inertia into output-side torque, $M_{t}$, $\&$ inertia, $J_{t}$. The speed is calculated from the output side to the input side and then integrated to an angle, $\theta_{t}$. There is also a speed proportional (viscous) friction in the transmission. On the output side the outputs torque $\&$ inertia are summed with the propeller shaft torque, $M_{p}$, $\&$ inertia, $J_{p}$, to produce the outputs speed, $\dot{\theta}_{p}, \&$ angle, $\theta_{p}$ using Newton's second law.

Note that no synchronizers are modeled and the model can not engage neutral gear. Therefore gear shifting will be instantaneous. This is an acceptable approximation when the clutch is disengaged, since the transmission input side has low inertia compared to the rest of the vehicle.

With the states $\dot{\theta}_{p}, \theta_{p}$, and $\theta_{t}$ the equations become:

$$
\begin{aligned}
M_{t} & =M_{c} i_{t, i} \\
\left(J_{t, i}+J_{p}\right) \ddot{\theta}_{p} & =M_{t}-b_{t} \dot{\theta}_{p}-M_{p} \\
\dot{\theta}_{t} & =\dot{\theta}_{p} i_{t, i}
\end{aligned}
$$

\subsection{Propeller Shaft}

The flexible propeller shaft is modeled in the same way as the clutch flexibility, (13). The equation for the propeller shaft is:

$$
M_{p}=c_{p}\left(\dot{\theta}_{p}-\dot{\theta}_{f}\right)+k_{p}\left(\theta_{p}-\theta_{f}\right)
$$

\subsection{Final Drive}

The final drive with differential is assumed to act symmetrically on the drive shafts. Therefore it can be modeled as the transmission but with fixed gear ratio, $i_{f}$, and inertia, $J_{f}$. With the states $\dot{\theta}_{d}, \theta_{d}$ the equations become:

$$
\begin{aligned}
\left(J_{p} i_{f}^{2}+J_{f}+J_{d}\right) \ddot{\theta}_{d} & =M_{p} i_{f}-b_{f} \dot{\theta}_{d}-M_{d} \\
\dot{\theta}_{f} & =\dot{\theta}_{d} i_{f}
\end{aligned}
$$

\subsection{Drive Shafts}

The flexible drive shafts can, with the symmetrical differential, be lumped into one and modeled in the same way as the clutch flexibility, (13). The equation is:

$$
M_{d}=c_{d}\left(\dot{\theta}_{f}-\dot{\theta}_{w}\right)+k_{d}\left(\theta_{f}-\theta_{w}\right)
$$

This is the main flexibility in the driveline.

\subsection{Vehicle Dynamics}

The non-driveline parts that affect the longitudinal dynamics are modeled in this block. That is, the wheels and vehicle. Tire dynamics are neglected and rolling condition is assumed. The wheels simply consists of a radius, $r_{w}$, an inertia, $J_{w}$ and a rolling resistance force, $F_{r}$.

Model inputs that directly affect the vehicle dynamics are braking force and road-slope angle, $\alpha$ (in radians). The road-slope angle is used to calculate the gradient force that is added with the braking force, rolling resistance and aerodynamic drag. The sign of the vehicle velocity is used so that rolling resistance, braking force and air resistance will oppose the vehicle movement.

The drag forces are subtracted from the drive shaft torque and divided by the vehicle mass, $m$, in order to calculate the vehicle acceleration, $a$, which is integrated to velocity and fed back to the drive shafts as angular velocity.

Discontinuity of Rolling Resistance The rolling resistance has a static component that changes sign with the velocity of the vehicle. Around zero velocity this leads to a large discontinuity in the rolling resistance. If this is not properly handled there can be significant oscillations in the vehicle velocity and loading torque. See Figure 2 for a simulation example of this. One possibility is to
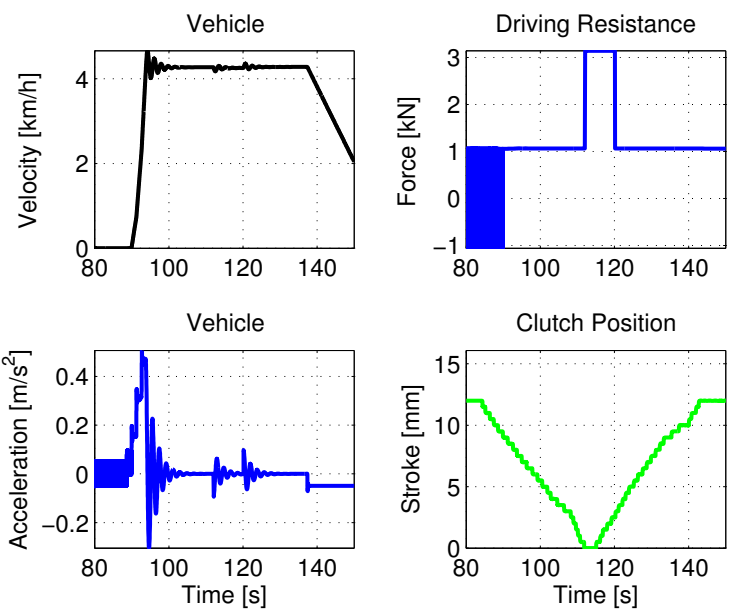

Fig. 2. In the upper right plot it is seen how the rolling resistance is constantly switching sign during the first ten seconds of the simulation. In the lower left plot it is seen in addition how the acceleration is switching, while the speed is practically zero, upper left plot.

use a state machine to control the states and transition between rolling and stand still. Another simpler cure to this problem is to smooth the rolling resistance using e.g. the following smoothing function

$$
f(v)=1-e^{-c_{s r} v^{2}}
$$

where $v$ is the vehicle speed (in $\mathrm{m} / \mathrm{s}$ ) and $c_{s r}$ is a tuning parameter. A smaller $c_{s r}$ gives more smoothing, which speeds up the simulation more but affects the rolling resistance higher up in the speed range. Here $c_{s r}=16$ is selected as an empirical value that gives large reductions in simulation time without a significant effect on the rolling resistance at speeds above $0.5 \mathrm{~km} / \mathrm{h}$, see Figure 3 . The simulation time was reduced with up to $85 \%$ when the smoothing function was used in cases similar to that in Figure 3.

With the states $v(\dot{v}=a)$ and $\theta_{w}$ the equations become:

$$
\begin{gathered}
F_{a}=\frac{1}{2} \rho_{a} c_{w} A_{f} v^{2}, \quad F_{g}=m g \sin (\alpha) \\
F_{r}=f(v)\left(c_{r 1}+c_{r 2}|v|\right) m g, \quad \dot{\theta}_{w}=v / r_{w} \\
\frac{M_{d}}{r_{w}}-\operatorname{sgn}(v)\left(F_{r}+F_{a}+F_{b}\right)-F_{g}= \\
=\left(m+\frac{J_{w}+J_{d}}{r_{w}^{2}}\right) a
\end{gathered}
$$



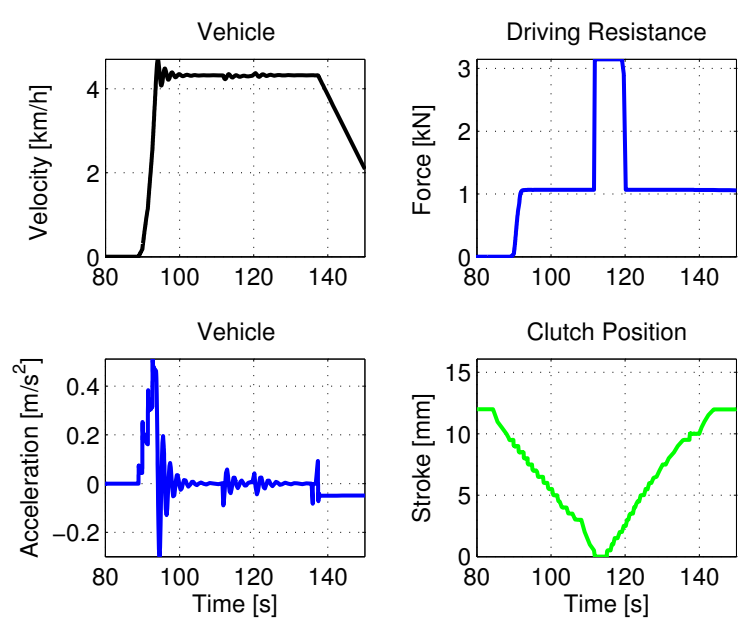

Fig. 3. Simulation with the smoothed rolling resistance model. In the upper right plot it is seen that the rolling resistance does not increase until the velocity starts to increase (upper left plot). Moreover the acceleration can be seen to be zero until movement starts. Moreover it can clearly be seen that when the driving resistance, due to discretization levels of road slope signal, makes steps, at $110 \mathrm{~s}$ and $120 \mathrm{~s}$, there are longitudinal oscillations.

\subsection{State-Space Model}

The vehicle speed, $y=v$, is the output and the velocities and torsions are chosen as state variables

$$
\begin{aligned}
x & =\left(x_{1}, x_{2}, x_{3}, x_{4}, x_{5}, x_{6}, x_{7}\right)^{T}= \\
& =\left(\omega_{e}, \theta_{e}-\theta_{t}, \omega_{p}, \theta_{p}-\theta_{f}, \omega_{d}, \theta_{d}-\theta_{w}, \omega_{w}\right)^{T}
\end{aligned}
$$

In order to get a compact representation of the model, the engine torque, $g_{1}=u_{1}=M_{e}$, and driving resistance, $g_{2}=F_{\mathrm{dr}}=F_{r}+F_{a}+F_{g}+F_{b}=F_{r}+F_{a}+u_{2}$, are collected into two separate signals. In the analysis in Section 3 the clutch is closed, gear is fixed, and velocity is positive. Then the system can be conveniently written as:

$$
\begin{aligned}
& \dot{x}=A x+B g(x, u) \\
& y=C x
\end{aligned}
$$

with,

$$
\begin{aligned}
g(x, u)= & {\left[u_{1}, \frac{\rho_{a} c_{w} A_{f} r_{w}^{2} x_{7}^{2}}{2}+\left(c_{r 1}+c_{r 2} r_{w} x_{7}\right) m g+u_{2}\right]^{T}, } \\
A= & \left(\begin{array}{ccccccc}
\frac{-c_{c}}{J_{1}} & \frac{-k_{c}}{J_{1}} & \frac{c_{c} i_{t}}{J_{1}} & 0 & 0 & 0 & 0 \\
1 & 0 & -i_{t} & 0 & 0 & 0 & 0 \\
\frac{c_{c} i_{t}}{J_{2}} & \frac{k_{c} i_{t}}{J_{2}} & \frac{-c_{1}}{J_{2}} & \frac{-k_{p}}{J_{2}} & \frac{c_{p} i_{f}}{J_{2}} & 0 & 0 \\
0 & 0 & 1 & 0 & -i_{f} & 0 & 0 \\
0 & 0 & \frac{c_{p} i_{f}}{J_{3}} & \frac{k_{p} i_{f}}{J_{3}} & \frac{-c_{2}}{J_{3}} & \frac{-k_{d}}{J_{3}} & \frac{c_{d}}{J_{3}} \\
0 & 0 & 0 & 0 & 1 & 0 & -1 \\
0 & 0 & 0 & 0 & \frac{c_{d}}{J_{4}} & \frac{k_{d}}{J_{4}} & \frac{-c_{d}}{J_{4}}
\end{array}\right) \\
J_{3}= & J_{p} i_{f}^{2}+J_{f}+J_{d}, \quad J_{4}=m_{v} r_{w}^{2}+J_{w}+J_{d} \\
c_{1}=c_{c} i_{t}^{2}+b_{t}+c_{p}, & c_{2}=c_{p} i_{f}^{2}+b_{f}+c_{d},
\end{aligned}
$$

$$
\begin{gathered}
B^{T}=\left(\begin{array}{ccccccc}
\frac{1}{J_{1}} & 0 & 0 & 0 & 0 & 0 & 0 \\
0 & 0 & 0 & 0 & 0 & 0 & \frac{-r_{w}}{J_{4}}
\end{array}\right) \\
C=\left(\begin{array}{llllllll}
0 & 0 & 0 & 0 & 0 & 0 & r_{w}
\end{array}\right)
\end{gathered}
$$

Note that (26) is a non-linear system as $g_{2}$ depends on $x_{7}^{2}$.

\subsection{Linearization}

The Bode diagram is used to analyze the gain and resonances in the system, and to facilitate this the system is linearized. Noting that the only non-linearity in the statespace model is in $g_{2}$ we can easily do the linearization by augmenting the $A$-matrix. This is done by doing the firstorder Taylor expansion around $v_{0}$ of the aerodynamic drag

$$
F_{a}=\frac{1}{2} \rho_{a} c_{w} A_{f}\left(2 v_{0} v-v_{0}^{2}\right)+\mathcal{O}\left(\left(v-v_{0}\right)^{2}\right)
$$

giving the addition

$$
\Delta a_{7,7}=\frac{-r_{w}^{2}\left(c_{r 2} m g-\rho_{a} c_{w} A_{f} v_{0}\right) v}{J_{4}}
$$

to element $a_{7,7}$ in $A$. The constant terms in the linearization are taken care of by the torque and torsions that correspond to steady state at $v_{0}$.

$$
\begin{aligned}
& \dot{x}_{\Delta}=A_{\text {aug }} x_{\Delta}+B u_{\Delta} \\
& y_{\Delta}=C x_{\Delta}
\end{aligned}
$$

were $A_{\text {aug }}$ is the $A$-matrix augmented with $\Delta a_{7,7}$.

\subsection{Model Validation}

The modular truck model has been validated using measurements from tip-in and tip-out maneuvers. Measurements of engine torque and road grade have been used as input for open-loop simulation of the model. Note that it is of great importance to have accurate road-grade information, when simulating the system. Otherwise the vehicle speed would have a large drift compared to the measurement. The model agrees well with measurement data for low gears, see Figure 4, and quite well for high gears, see Figure 5. In the latter case there were some small high frequency oscillations that were not captured in the model. Nonetheless the main vehicle shuffle frequency and amplitude have been captured in both cases. Therefore the model is suitable for simulations of cases where vehicle shuffle is a concern.
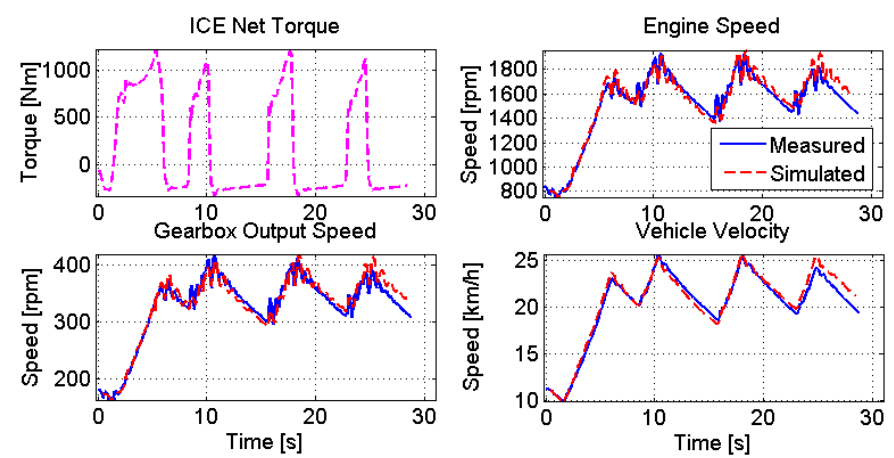

Fig. 4. Model validation using tip-in/tip-out maneuvers for fifth gear. The model is run in open loop and it agrees well with data. 

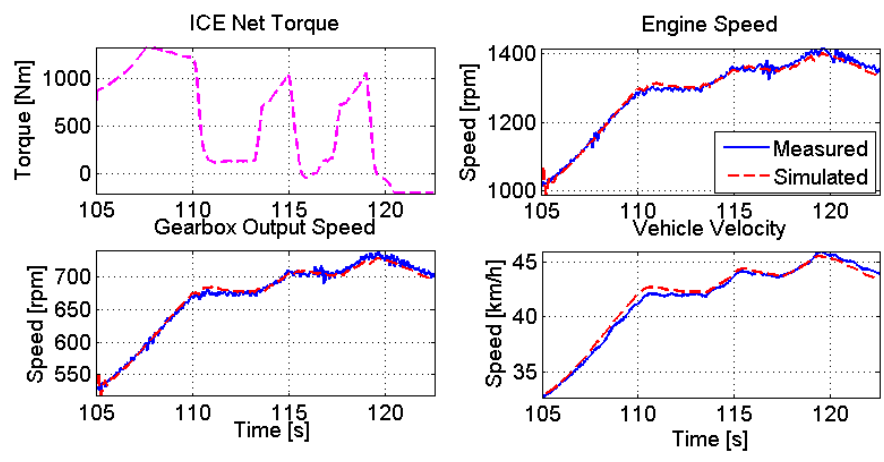

Fig. 5. Model validation using tip-in/tip-out maneuvers for ninth gear. The model is run in open loop and it captures the trends in speed variations as well as the shuffle oscillations well. There are some quick oscillations that are missed.

\section{SLOPE SIGNAL EFFECT ON SIMULATION}

The linearized state-space model (30) is used to investigate the effect of a discretized slope signal on vehicle shuffle. First, no braking is assumed. Second, for slopes less than $20 \%, \sin (\alpha)$ can, with great accuracy, be considered equal to the road slope in percent $(=\tan \alpha)$. Since roads made for heavy duty trucks in Sweden have a maximum slope of $12 \%$ (highways have a maximum of $8 \%$ slope), (Swedish Road Administration, 2004), this is a very sound simplification. These two assumptions make $u_{\Delta, 2}$ proportional to the road slope, and therefore suitable as input signal for this study.

The truck has been simulated, in first gear with constant engine torque, using a measured driving resistance as input. The measured driving resistance contains three pulses, one long and two short, see Figure 6 . The changes in driving resistance are due to that the road-slope signal is discretized in amplitude. This kind of signal contains a very wide frequency range. Therefore it induces oscillations in the driveline that are visible alongside the ramp changes in velocity. This can be explained by viewing the Bode plot of the system found in Figure 7. In the bode plot, there is a resonance peak in the transfer function from $F_{\mathrm{dr}}$ to $v$. This peak is the reason for the oscillations, however the peak has a low amplitude compared to the low frequency gain. Therefore the ramp changes in velocity are much greater than the oscillations. Note that also the transfer function from $M_{e}$ to $v$ has a resonance peak that can result in driveline oscillations. Consequently if the system is under feedback, e.g. speed control, then both resonance peaks can be excited by a slope step, leading to even more driveline oscillations.

To highlight this a PI-controller for engine speed control is introduced

$$
\begin{aligned}
e & =\dot{\theta}_{\mathrm{e}, \mathrm{ref}}-\dot{\theta}_{e} \\
\dot{x}_{8} & =e \\
M_{e} & =K_{P} e+K_{I} x_{8}
\end{aligned}
$$

where $K_{P}$ and $K_{I}$ are the controller parameters. With the speed controller the results in Figure 7 and Figure 8 are obtained. The driveline oscillations are now more clearly seen as well as the resonance peak in the transfer function from $F_{\mathrm{dr}}$ to $v$, which now also attains its maximum at the resonance. A better tuning or a more advanced controller could perhaps avoid the oscillations in the closed loop case, but this would only cure the symptoms and not the cause, which is the high frequency content in the input. It can thus be concluded that some kind of filtering should be applied to the road slope signal.

It should however be noted that the occurrence of the sharp resonance peak, and therefore the need for filtering, is dependent on the selected gear. In the closed loop case, higher gear means that the peak in the transfer function moves towards lower frequencies, while in the open loop case it moves slightly towards higher frequencies. The peak also becomes less pronounced with higher gear numbers and vanishes at gear eight. So no filtering is needed for gears higher than seven.
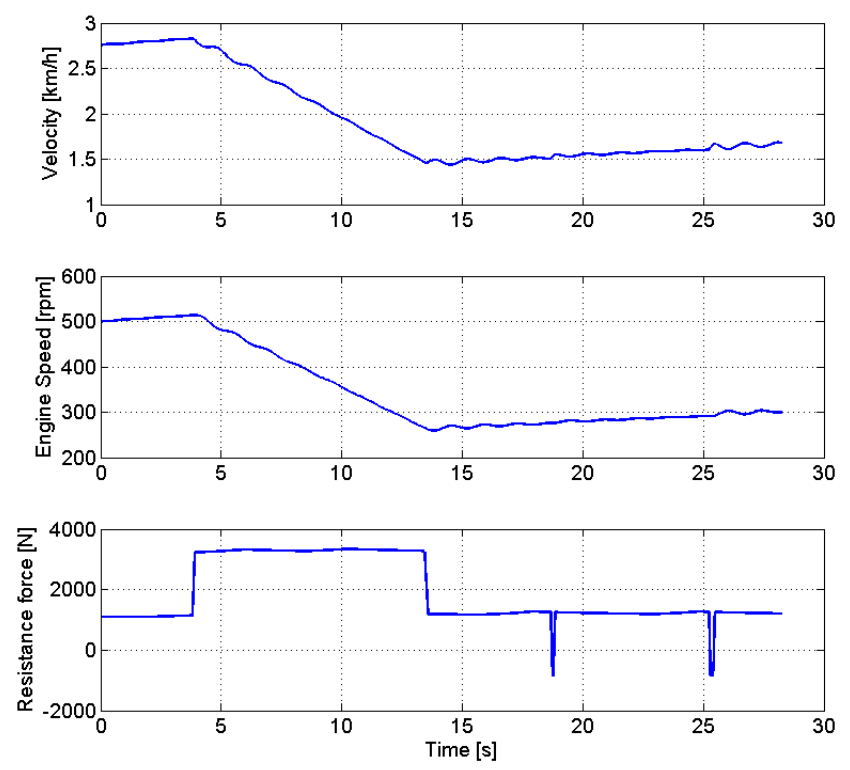

Fig. 6. Showing how a one percentage point step in road slope affects truck behavior in $1^{\text {st }}$ gear when engine torque is constant. A change in vehicle velocity with an oscillation superimposed can be seen.

\section{ROAD FREQUENCY}

When filtering the road-slope signal, it is of great interest to keep the important information in the signal. In order to achieve this it must be understood how a road-slope profile typically looks like.

According to official road-design policies (see Swedish Road Administration (2004) for Swedish and AASHTO (2004) for US), the road segments between constant slopes have a parabolic shape,

$$
z=\frac{x^{2}}{2 R}
$$

where $z$ is the elevation, $x$ the longitudinal position and $R$ the curve parameter. The parabola works as an approximation of a circle with radius $R$. For Swedish highways ( $89 \mathrm{~km} / \mathrm{h}$ for heavy duty trucks) $R$ can be as low as $1250 \mathrm{~m}$ and in cities $(50 \mathrm{~km} / \mathrm{h}) R$ can be as low as $300 \mathrm{~m}$. The slope can be calculated as, 

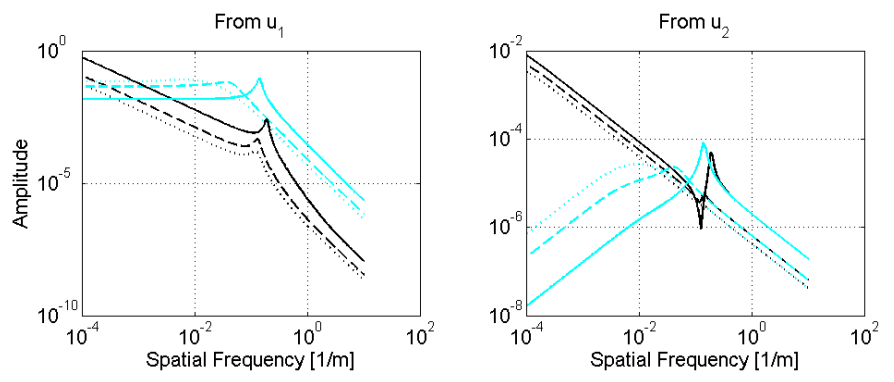

Fig. 7. Bode plot of the open-loop system (black) and the closed-loop system (cyan), both systems in first (solid), sixth (dashed), and eighth (dotted) gear. Spatial frequency is used in order to enable comparisons with the road spectra in Section 4. The spatial frequency has been calculated using the maximum speed of each gear. For the open loop system $u=\left[M_{e} F_{\mathrm{dr}}\right]^{T}$ and for the closed loop system $u=\left[w_{\mathrm{e}, \text { ref }} F_{\mathrm{dr}}\right]^{T}$. For both cases $y=v$. It can be seen that the resonance peaks vanishes at eighth gear. For the closed loop case, in the right plot, the peak moves towards lower frequencies as the gear number is increased.
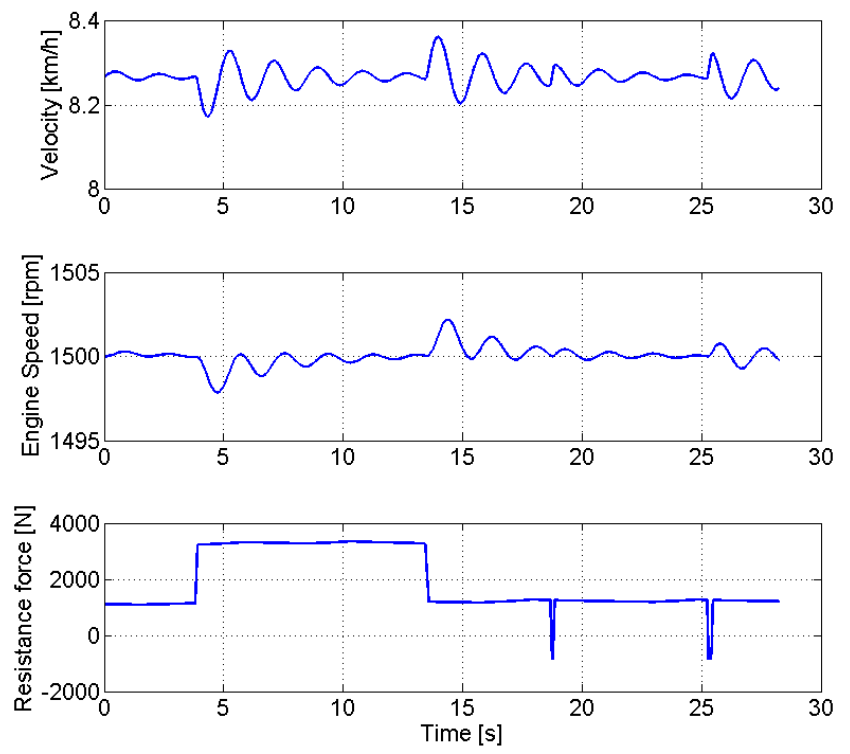

Fig. 8. Oscillations in vehicle speed (longitudinal shuffle) can be seen when one-percentage-point pulses in road slope are present. Engine speed is kept constant by a PI-controller.

$$
\begin{aligned}
\tan \alpha=\lim _{x_{2} \rightarrow x_{1}}\left(\frac{z_{2}-z_{1}}{x_{2}-x_{1}}\right) & =\lim _{x_{2} \rightarrow x_{1}}\left(\frac{x_{2}^{2}-x_{1}^{2}}{2 R\left(x_{2}-x_{1}\right)}\right)= \\
= & \lim _{x_{2} \rightarrow x_{1}}\left(\frac{x_{2}+x_{1}}{2 R}\right)=\frac{x}{R}
\end{aligned}
$$

As stated earlier, slopes can be considered small and together with $x \approx v t$ it yields,

$$
\alpha=\frac{v}{R} t
$$

This means that the slope varies as a piece-wise linear and continuous function. A piece-wise linear function contains sharp knees and has an infinite frequency content but the amplitude decreases with frequency. To get an idea of the spectrum a synthetic road is constructed by picking a number of random inclinations with random lengths, then connecting these slopes with vertical curves of random radii. For simplicity reasons uniform distributions are assumed for all variables. The inclinations have been selected from the range dictated by Swedish Road Administration (2004). The radii have been selected from a narrow range above the minimum value. The range of the slope-length distributions are set exponentially with respect to the inclination, with flat ground giving the widest range. This is to mimic that in reality flat segments usually are longer then steep slopes. These assumptions are used to construct an approximation of a worst-case road. The resulting frequency spectrum is seen in Figure 9. Most of the power is in spatial frequencies below $3 \cdot 10^{-4} 1 / \mathrm{m}$.

This theoretical "worst-case road" has been complemented with frequency spectra of different measured road segments ${ }^{1}$ that indicate similar cut-off frequencies and rolloff rates, Figure 9. This indicates that the synthetisation method is sound and therefore it has been used for making a city road (50 km/h speed limit). The different limitations on maximum slope and $R$ results in a road with higher frequency content. The main power is below $1 \cdot 10^{-3} 1 / \mathrm{m}$.

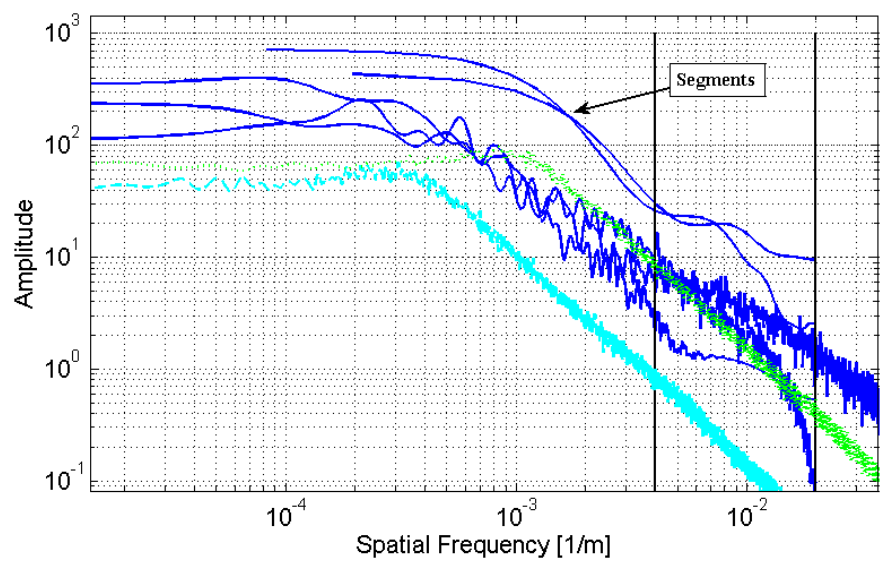

Fig. 9. Frequency spectra for five measured highways (blue solid), one synthetic highway (cyan dashed), and one synthetic city road (green dotted). They all have similar shapes and most of their energy in frequencies below $1 \cdot 10^{-3} 1 / \mathrm{m}$. The two shorter curves correspond to segments of the longer roads. The two black vertical lines mark the cut-off frequency and the $-30 \mathrm{~dB}$ frequency of the filter.

Looking at the data for the road slope frequency content, Figure 9, and the Bode diagram for the model, Figure 7, one sees that the road data starts to fall off for frequencies below the resonance frequency of the driveline. This provides an opportunity to reduce the problem of oscillations by filtering the road slope signal. Especially since the Bode diagram represents the case with lowest spatial frequency (highest speed for each gear), which represents the worst case with minimum separation between frequencies.

\footnotetext{
1 The road segments are the Järna segment, Jönköping-Linköping,
} Koblenz-Trier, Norrköping-Södertälje and the Olstorp segment 

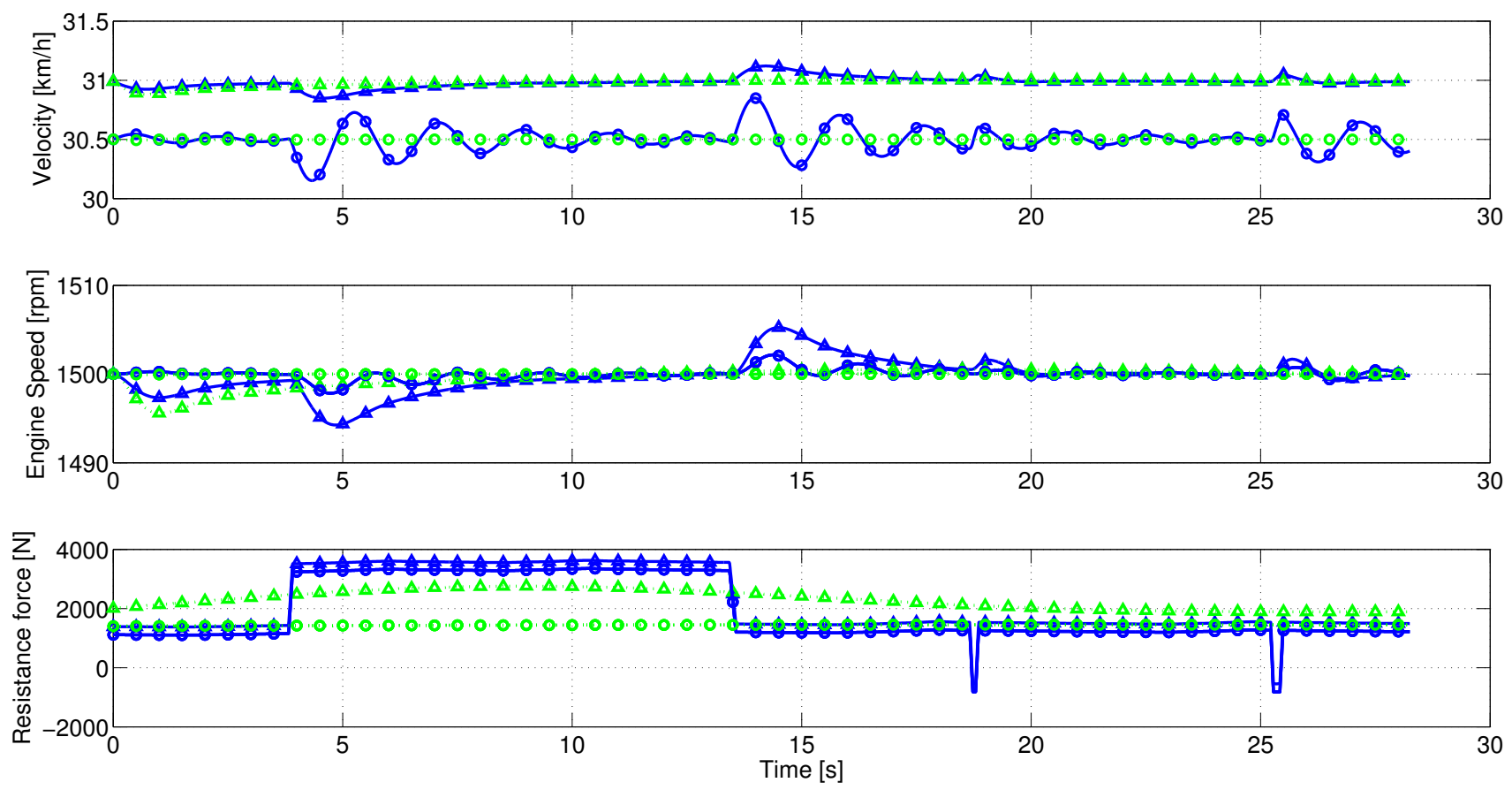

Fig. 10. Original slope signal with its simulation response in solid blue. The acausally filtered signal and its response are shown in dashed green. Triangles are seventh gear and circles are first gear. The velocity response in first gear has been scaled. The oscillations are successfully filtered. Note that in first gear the slope signal is filtered more since the pulses are shorter in spatial coordinates.

\section{FILTER DESIGN}

The design rules for the filter are that it should have little impact on the naturally occurring road frequencies and damp those that could excite driveline oscillations.

According to the investigation in the previous section the important road frequencies lie below $1 \cdot 10^{-3} 1 / \mathrm{m}$, the location of the knee of the synthetic city road. However note that the two shorter segments in Figure 9 have a later roll off than the long roads because these particular segments are rich on high frequency content. Likewise choosing the filter cut-off frequency at the knee of the city road profile might lead to filtering of naturally occurring frequencies on a segment of this profile. Therefore the cutoff frequency is taken with one decade of margin compared to the knee, i.e $f_{\text {co }}=4 \cdot 10^{-3} 1 / \mathrm{m}$. However there is never any guarantee that no real frequencies will be filtered as the theory have shown an infinite frequency content.

Frequencies that need to be damped are those around the resonance peak, see Figure 7, that exists for gear seven or lower. Maximum speed in seventh gear is about $50 \mathrm{~km} / \mathrm{h}$ so it makes sense to choose $f_{\text {co }}$ from the city road. The lowest frequency for any resonance peak is $f_{\mathrm{lo}}=2 \cdot 10^{-2} 1 / \mathrm{m}$, which is that of gear seven in closed loop. As the resonance peak is quite low in seventh gear, $-30 \mathrm{~dB}$ of attenuation is chosen at $f_{\text {lo. }}$. The filter will naturally attenuate more at the peaks for lower gears.

These requirements can be fulfilled by, for example a third or higher order low pass Butterworth filter with a cut-off frequency of $4 \cdot 10^{-3} 1 / \mathrm{m}\left(-40 \mathrm{~dB}\right.$ at $\left.f_{\text {lo }}\right)$. Note that while on one hand higher order gives a steeper gain curve and more margin to the requirements, on the other it gives more phase lag, i.e. time delay. In addition a higher filter order is more complex so computation time will increase. Therefore the lowest possible order is desirable in an online application. It might also be desirable to filter in the time domain, in that case the filter will be speed dependent. In an off-line application computational time is of less importance and the signal can be filtered both in the forward and reverse direction in order to get a zero phase filter.

\section{RESULTS}

Figure 10 shows that third order filtering of driving resistance effectively eliminates the oscillations in both first and seventh gear. In Figure 11 it can be seen that the high frequency content in the road slope sensor is significantly reduced whereas the real road profile is essentially the same. A road profile together with causally and non-causally filtered versions are displayed in Figure 12, showing that the general behavior of the road profile is preserved. It can thus be concluded that the filter design works as intended. Furthermore a third order filter is sufficient even in the off-line application, where it would be trouble free to increase the order.

\section{CONCLUSIONS}

In this paper a model that describes vehicle shuffle of a heavy duty truck has been summarized and validated. Special emphasis was given to the interaction between driveline and road. In particular it was shown that smoothing the rolling resistance around zero speed gives a significant reduction in simulation time for driving with start and stop scenarios. 


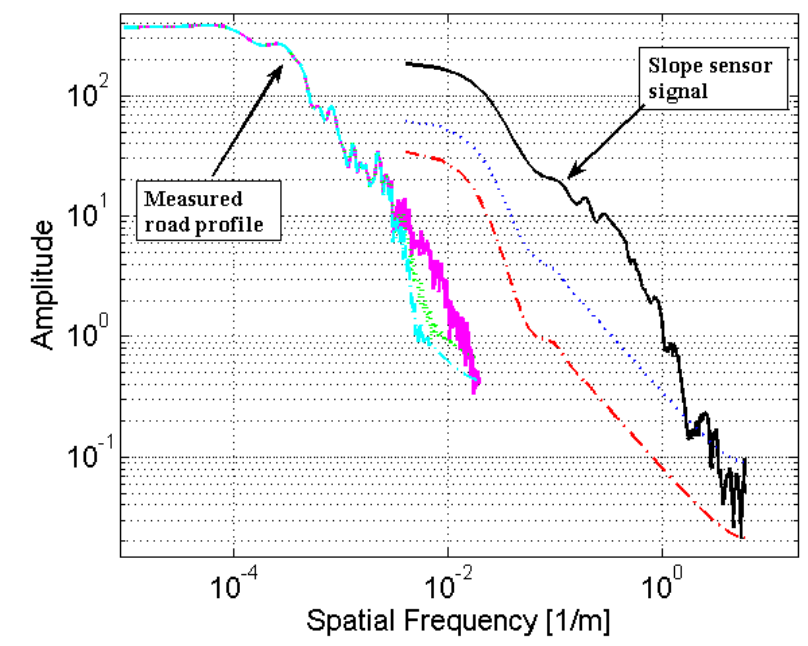

Fig. 11. A real road profile in solid. With its filtered ditos in dashed-doted (non-causal) and dotted (causal) slightly below (hard to tell apart). The short measured slope signal is also found in solid. It too has its filtered dito in dashed-doted (non-causal) and dotted (causal) below. The measured signal has lost a lot of its high frequency content whereas the road profile has kept its low frequency content. Just as intended.

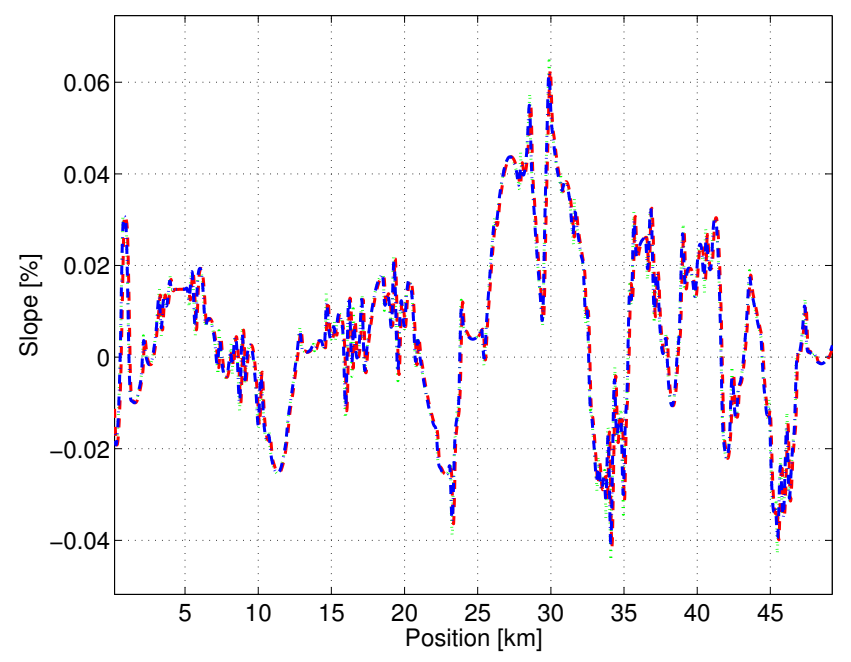

Fig. 12. The road profile as function of position. Unfiltered in dotted green, causally filtered in dashed red and non-causally filtered in dash-dotted blue. The three signals are practically the same.

Furthermore, experimental data from a production road slope sensor was shown to have a big impact on the simulation of the vehicle. In particular the high frequency content, introduced by amplitude discretization in the sensor, was shown to excite driveline oscillations. The oscillations also become more pronounced when the driveline had a feedback speed controller.

The frequency content in a theoretical worst-case road and measured roads, have been investigated and found to be below the resonance frequency of the model in every gear. It was also shown that the frequency separation was large enough to make a low-pass-filter design possible for the road-slope sensor.
Finally a design is proposed for a filtering method consisting of a low pass Butterworth filter in the spatial domain that eliminates erroneous behavior caused by high frequency disturbances in the slope signal, e.g. discretization. Applying the filter to measured data shows that the driveline oscillations are significantly attenuated, while the shape of the road-slope profile is maintained.

\section{REFERENCES}

AASHTO (2004). A policy on geometric design of highways and streets: 2004. American Association of State Highway and Transportation Officials.

Bae, H.S., Ryu, J., and Gerdes, J.C. (2001). Road grade and vehicle parameter estimation for longitudinal control using gps. In 2001 IEEE Intelligent Transportation Systems.

Dolcini, P., de Wit, C.C., and Béchart, H. (2008). Lurch avoidance strategy and its implementation in AMT vehicles. Mechatronics, 18(1), 289-300.

Fredriksson, J. and Egardt, B. (2003). Active engine control for gear shifting in automated manual transmissions. Int. J. of Vehicle Design, 32(3-4), 216-230.

Gao, B., Lei, Y., Ge, A., Chen, H., and Sanada, K. (2011). Observer-based clutch disengagement control during gear shift process of automated manual transmission. Vehicle System Dynamics, 49(5), 685-701.

Garofalo, F., Glielmo, L., Iannelli, L., and Vasca, F. (2002). Optimal tracking for automotive dry clutch engagement. In 2002 IFAC, 15th Triennial World Congress.

Gerhardt, J., Hönninger, H., and Bischof, H. (1998). 980801: A new approach to functional and software structure for engine management systems - bosch me7. In SAE 1998 International Congress and Exposition.

Lingman, P. and Schmidtbauer, B. (2002). Road slope and vehicle mass estimation using kalman filtering. Supplement to Vehicle System Dynamics, 37(1), 12-23.

Moon, S., Kim, M., Yeo, H., Kim, H., and Hwang, S. (2004). Design and implementation of clutch.by.wire system for automated manual transmissions. International Journal Vehicle Design, 36(1), 83-100.

Myklebust, A. and Eriksson, L. (2012). Torque model with fast and slow temperature dynamics of a slipping dry clutch. In 2012 IEEE Vehicle Power and Propulsion Conference.

Pettersson, M. (1997). Driveline Modeling and Control. Ph.D. thesis, Linköpings Universitet.

Pettersson, M. and Nielsen, L. (2000). Gear shifting by engine control. IEEE Transactions Control Systems Technology, 8(3), 495-507.

Pettersson, M. and Nielsen, L. (2002). Diesel engine speed control with handling of driveline resonances. Control Engineering Practice, Advances in Automotive Control, 11(3), 319-328.

Sahlholm, P. (2011). Distributed road grade estimation for heavy duty vehicles. Ph.D. thesis, KTH School of Electrical Engineering.

Sebsadji, Y., Glaser, S., Mammar, S., and Dakhlallah, J. (2008). Road slope and vehicle dynamics estimation. In 2008 American Control Conference.

Swedish Road Administration (2004). Vägar och gators utformning - linjeföring. Technical report, Vägverket Publikation 2004:80. 